\title{
Calculation of electron mobility and effect of dislocation scattering in GaN
}

\author{
Janardan Kundu ${ }^{1}$, C.K. Sarkar ${ }^{2}$ and P.S. Mallick ${ }^{1}$ \\ ${ }^{1}$ Department of Electronics and Communication Engineering, National Institute of Science and Technology, \\ Palur Hills, Berhampur 761 008, India \\ E-mail:psmallick@yahoo.com \\ ${ }^{2}$ Department of Electronics and Telecommunication Engineering, Jadavpur University, \\ Kolkata 700 032, India
}

\begin{abstract}
The electron mobility of GaN has been obtained at various temperatures by the relaxation time approximation method. The effect of dislocation scattering has also been discussed and calculated alongwith other important scattering mechanisms in this material. The results agree with other available experimental and theoretical data.
\end{abstract}

Keywords: electron mobility, dislocation scattering, gallium nitride.

Manuscript received 31.12.06; accepted for publication 26.03.07; published online 01.06.07.

\section{Introduction}

Gallium nitride, a direct bandgap semiconductor, has emerged as an important material for high-power, optoelectronic as well as for high temperature devices because of its large bandgap $(3.4 \mathrm{eV})$, strong bond strength $(2.3 \mathrm{eV} /$ bond $)$ and high breakdown voltage $\left(3 \times 10^{6} \mathrm{~V} / \mathrm{cm}\right)[1]$. Recently the material has become more popular because of several new applications including blue light emitting diodes and blue laser diodes [2]. GaN is normally grown either by metalorganic chemical vapor deposition (MOCVD), molecular beam epitaxy (MBE) or hybrid vapor phase epitaxy (HVPE) on sapphire $\left(\mathrm{Al}_{2} \mathrm{O}_{3}\right)$ or $\mathrm{SiC}$ substrate with large lattice mismatch. The most commonly used substrate is $\mathrm{Al}_{2} \mathrm{O}_{3}$ with $13.8 \%$ lattice mismatch and $\mathrm{SiC}$ substrate with $4 \%$ lattice mismatch. The large lattice mismatch with the substrate produces large amount of dislocation at the interfacial layer resulting very poor interface characteristic. As we move away from the interfacial layer, the dislocation density decreases very fast. This suggests that whole GaN epilayer consists of two layers which was also suggested by D.C. Look et al. [3]. In order to calculate the mobility in $n$-type GaN, we have considered the two-layer model. For bulk layer away from the interface, the dominant scattering mechanism is considered to be acoustic phonon scattering via deformation potential, piezoelectric coupling and the non-phonon scattering such as ionized impurity scattering and the neutral impurity scattering. On the contrary, the dominant scattering mechanism near the interfacial region is assumed to be dislocation scattering only. The electrical transport properties of the entire GaN epilayer would be influenced by the dislocation scattering dominant near the interfacial region. In this paper, the two-layer model of $\mathrm{GaN}$ has been considered for theoretical calculation of electron mobility. Effect of dislocation scattering in the interfacial layer has been calculated and clarified.

\section{Theory}

The electron mobility considering various scattering mechanisms can be given by solving the Boltzmann equation in the relaxation time approximation as

$$
\mu=\frac{e\langle\tau\rangle}{m^{*}}
$$

where $\langle\tau\rangle$ is the average relaxation time over the electron energies and $\mu$ is the mobility, and $m$ is the effective mass of electron. In the following sections, the expressions of relaxation time and mobility caused by different scattering mechanisms have been given.

\section{Ionized impurity scattering}

The amount of scattering due to electrostatic forces between the carrier and the ionized impurity depends on the interaction time and the number of impurities. Larger impurity concentrations result in a lower mobility [8]. The standard formula for calculating the average relaxation time is given by 


$$
\left\langle\tau_{i i}(\varepsilon)\right\rangle=\frac{e}{m^{*}} \frac{\int_{0}^{\infty} \tau_{i i}(\varepsilon) \varepsilon^{3 / 2} \frac{d f_{0}}{d \varepsilon} d \varepsilon}{\int_{0}^{\infty} \varepsilon^{3 / 2} \frac{d f_{0}}{d \varepsilon} d \varepsilon}
$$

The mobility associated with ionized impurity scattering has been calculated as

$\mu_{i i}=\frac{128 \sqrt{2 \pi}^{1 / 2} \epsilon^{2}(k T)^{3 / 2}}{N_{I} Z^{2} e^{3} m^{* 1 / 2}[\ln (1+y)-y /(1+y)]}$,

where $y=\frac{24 \in m^{*}(K T)^{2}}{\hbar^{2} e^{2} n}$.

\section{Neutral impurity scattering}

When an electron passes close to neutral atom, momentum can be transferred through a process in which the free electron exchanges with a bound electron on the atom. The relaxation time can be written as [5]

$$
\tau_{n i}(\varepsilon)=\frac{m^{*}}{20 N_{n} \hbar a_{0}} .
$$

The mobility associated with the neutral impurity scattering has been calculated as

$\mu_{n i}=\frac{e}{20 N_{n} \hbar a_{0}}=\frac{e^{3} m^{*}}{80 \pi N_{n} \hbar^{3} \epsilon}$,

where $a_{0}$ is the effective Bohr radius of donor, and $N_{n}$ is the concentration of neutral impurities.

\section{Acoustic phonon: deformation potential scattering}

The acoustic mode lattice vibration induced changes in lattice spacing, which change the bandgap from point to point. Since the crystal is 'deformed' at these points, the potential is called the deformation potential. The corresponding relaxation time can be written as [6]

$\tau_{d p}(\varepsilon)=\frac{\pi \hbar^{4} \rho s^{2}}{\sqrt{2} E_{1}^{2} m^{* 3 / 2}(k T)} \varepsilon^{-1 / 2}$,

where $\rho$ is the crystal density, $S$ is the average velocity of sound, and $e_{1}$ is the deformation potential. Here, $\rho \mathrm{s}^{2}$ $=c_{1}$ is the longitudinal elastic constants.

The mobility associated with the deformation potential scattering has been calculated as

$$
\mu_{d p}=\frac{e\left\langle\tau_{d p}\right\rangle}{m^{*}}=\frac{2 \sqrt{2} \pi^{1 / 2} \hbar^{4} \rho s^{2} e}{3 E_{1}^{2} m^{* 5 / 2}(k T)^{3 / 2}} .
$$

For $\mathrm{GaN}$ the acoustic deformation potential is equal to $9.2 \mathrm{eV}$ [4].

\section{Acoustic phonon: piezoelectric potential scattering}

A relaxation time can be defined for the piezoelectric potential mechanism because the energy change during the collision is small. The relaxation time is [6]

$\tau_{p e}(\varepsilon)=\frac{2 \sqrt{2} \pi \hbar^{2} \in}{e^{2} p^{2} m^{* 1 / 2}(k T)} \varepsilon^{1 / 2}$,

where $p=\left[\frac{h_{p z}^{2}}{\rho s^{2} \epsilon}\right]$ is the piezoelectric coupling coefficient, $h_{p z}$ is the piezoelectric constant. The mobility associated with the piezoelectric potential scattering has been calculated as

$\mu_{p e}=\frac{e\left\langle\tau_{p e}(\varepsilon)\right\rangle}{m^{*}}=\frac{16 \sqrt{2} \pi^{1 / 2} \in h^{2}}{3 e P^{2} m^{* 3 / 2}(k T)^{1 / 2}}$.

At $300 \mathrm{~K}$, the piezoelectric potential scattering rate is about five times smaller than the deformation potential rate [4].

\section{Optical phonon: polar scattering}

For this scattering, a relaxation time solution to the Boltzmann equation is not possible. Since $\tau$ becomes a function of the perturbation strength itself instead of just the energy $\varepsilon$ of electrons. The relaxation time

$$
\tau_{p o}(\varepsilon)=2^{3 / 2} \pi \frac{\hbar^{2}\left(e^{T_{D} / T}-1\right) \chi\left(T_{D} / T\right)}{e^{2}\left(k T_{D}\right) m^{* 1 / 2}\left(\epsilon_{\infty}^{-1}-\epsilon^{-1}\right)} \varepsilon^{1 / 2} .
$$

The corresponding mobility calculated as

$\mu_{p o}=\frac{2^{9 / 2} \pi^{1 / 2} \hbar^{2}(k T)^{1 / 2}\left(e^{T_{D} / T}-1\right) \chi\left(T_{D} / T\right)}{3 e\left(k T_{D}\right) m^{* 3 / 2}\left(\epsilon_{\infty}^{-1}-\epsilon^{-1}\right)} \varepsilon^{1 / 2}$.

The mobilities limited by the lattice phonons (including polar optical phonon, acoustic-mode deformation potential and piezoelectric potential) scattering are independent of impurity levels, so their temperature dependence is universal for GaN [4].

\section{Dislocation scattering}

One of the biggest problems of $\mathrm{GaN}$ is the lack of a lattice-matched substrate, since bulk GaN is very difficult to grow in large sizes. Thus, epitaxial growth of $\mathrm{GaN}$ on $\mathrm{Al}_{2} \mathrm{O}_{3}$, which has a $14 \%$ lattice mismatch and $34 \%$ mismatch in the thermal expansion coefficient. Due to the large lattice mismatch of $\mathrm{GaN}$ with the substrates, on which it is epitaxially grown ( $\mathrm{SiC}$ and sapphire), dislocations are typically formed. The relaxation time is 
$\tau=\frac{8\left(\in \in_{0}\right)^{2} a^{2} m^{* 2}}{N e^{4} f^{2} L_{D}}\left(v_{t}^{2}+\hbar^{2} / 4 m^{* 2} L_{D}^{2}\right)^{3 / 2}$,

where $V_{t}$ is the component of $V$ perpendicular to dislocation line, $a$ is the distance between imperfection centers along the dislocation line, and $f$ is their occupation probability.

Averaging with the equilibrium distribution function, the mobility calculated as

$$
\mu=\frac{30 \sqrt{2 \pi}\left(\varepsilon \varepsilon_{0}\right)^{2} a^{2}\left(K_{\mathrm{B}} T\right)^{3 / 2}}{e^{3 f^{2}} L_{D} m^{* 1 / 2} N} m .
$$

Since the reciprocal values of the relaxation time resulting from different physical mechanism are additive, the scattering caused by the charge of dislocation in $n$-type semiconductors gives the dominant effect below the room temperature [7].

\section{Results and discussion}

The theoretical results for mobilities including different types of scattering mechanisms such as ionized impurity, neutral impurity, acoustic phonon via potential deformation, piezoelectric, polar optical phonon and dislocation scattering at different temperatures have been calculated using the parameters of $n$-type GaN. The dislocation density is $10^{15} \mathrm{~m}^{-2}$ and the carrier concentrations in bulk and interfacial layers are taken as $1.3 \times 10^{17}$ and $7 \times 10^{24} \mathrm{~m}^{-3}$, respectively.

Fig. 1 represents the variation of electron mobility with temperature for various types of scattering mechanisms such as ionized impurity, neutral impurity, acoustic phonon via deformation potential, piezoelectric scattering, polar optical phonon scattering and dislocation scattering individually.

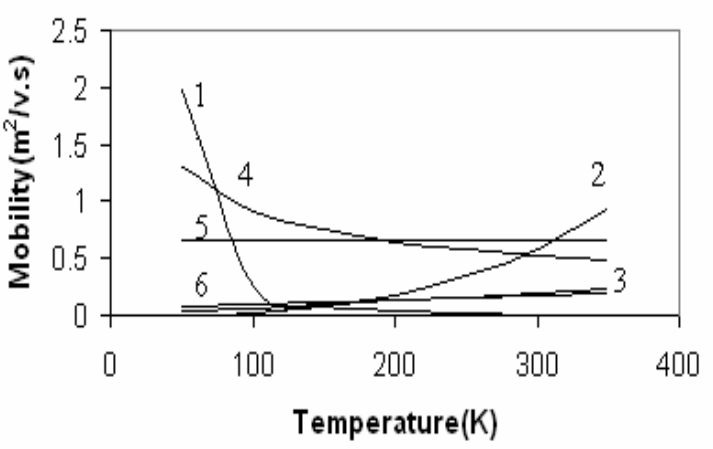

Fig. 1. Variation of electron mobility with temperature for acoustic phonons via deformation potential (1), ionized impurity (2), polar optical phonon (3), piezoelectric potential (4), neutral impurity (5) and dislocation scatterings (6).

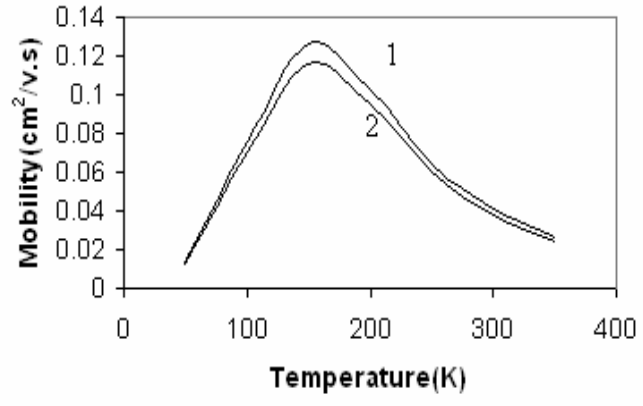

Fig. 2. Variation of electron mobility with temperature in twolayer model considering without (1) and with (2) the dislocation scattering.

From Fig. 2, we can conclude that the influence of the dislocation scattering on the mobility of $\mathrm{GaN}$, compared to different lattice scatterings becomes significant at a high density of dislocations at the interfacial layer of $\mathrm{GaN}$ above $10^{9} \mathrm{~cm}^{-2}$.

\section{Conclusion}

We have calculated the combined electron mobility of $\mathrm{GaN}$ with two-layer model by considering the various scattering mechanism such as ionized impurity, neutral impurity, potential deformation, piezoelectric potential, polar optical phonon and dislocation scattering. In Fig. 2, we have shown the effect of dislocation scattering in the mobility analysis of GaN with two-layer model, and we found that the dislocation scattering plays a dominant role in mobility analysis when the dislocation density is high and can indeed directly affect mobility. From the above results, we can conclude that the agreement between measured and calculated mobility is better at the room temperature than at lower temperatures. This can be explained by decreasing proportion of ionized electrons in $\mathrm{GaN}$ at lower temperature. Overall the calculated mobility at the room temperature and at $150 \mathrm{~K}$ agrees quite satisfactorily with the published theoretical and experimental results [6-8].

\section{References}

1. S.C. Jain, M. Willander, J. Narayan and R. Van Overstraeten, III-nitrides: growth, characterization and properties // J. Appl. Phys. 87, No. 3, p. 965-1006 (2000).

2. S. Nakamura, S.J. Pearton and G. Fasal, The blue LASER diodes. Springer, Berlin, 2000.

3. D.C. Look, J.R. Sizelove, Dislocation scattering in GaN // Phys. Rev. Lett. 82, No. 6, 1999.

4. D.C. Look, Electrical characterization of GaAs materials and devices. Wiley, New York, 1989.

5. C. Erginsoy // Phys. Rev. 79, p. 1013 (1950).

6. D.A. Anderson and N. Apsley // Semiconductor Science and Technology 1, June 9 (1986).

7. B. Podor // Phys. status solidi 16, p. k167 (1966).

8. Subhabrata Dhar and Subhasis Ghosh // J. Appl. Phys. 86 (5), 1st Sept. (1999). 